\title{
Why Is The Identification of Causative Agent of Nocardiosis Essential at The Species Level?
}

\author{
Masoud Keikha ${ }^{*}$ \\ ${ }^{1}$ Department of Microbiology, School of Medicine, Isfahan Medical University, Isfahan, Iran
}

Received Sep 02, 2017; Accepted Oct 25, 2017

\begin{abstract}
Nocardia is a genus of weakly gram-positive, filamentous, aerobic, relatively slow-growing and partially acid-fast bacteria. These bacteria are ubiquities in environmental resources such as water, soil, dust, decomposing animal feces, and vegetables. This group of bacteria can enter the human body through inhalation and traumatic cutaneous inoculation and cause Nocardia infections. There are various types of nocardiosis including pulmonary, brain, cutaneous, cutaneous-lymphatic, and ocular infections. These infections often occur in immunocompromised patients including organ transplant recipients, corticosteroid drugs consumers, human immunodeficiency virus (HIV) patients, cancerous and even healthy individuals [1-3].
\end{abstract}

Identification of the Nocardia species is necessary due to differences in the pattern of drug susceptibility of species and for epidemiological studies as well [4]. These infections are often misdiagnosed with the viral infection, mycoplasma, fungi, cancer, and tuberculosis. Reports from Iran shows an incidence rate of about $1.88 \%$ [3]. Phenotypic (conventional) methods and molecular techniques are commonly used to identify the Nocardia species. Conventional diagnosis is based on colony morphology, Gram and Kinyoun stains, resistance to lysozyme broth, ability to grow at $45^{\circ} \mathrm{C}$, decomposition of casein, xanthine, hypoxanthine, esculin, adenine and gelatin, and utilization of citrate, acetamide, and carbohydrates as a sole source of carbon and nitrogen. This approach is often costly and time-consuming and requires trained technicians, yet leads to the wrong diagnosis in $37 \%$ of cases [4]. The current molecular methods such as PCR amplification of 16S rRNA and Heat Shock Protein gene followed by restriction fragment length polymorphism (PCR-RFLP), and sequencing of the conserved regions of $16 \mathrm{~S}$ rRNA, hsp65, gyrB and sodA genes have proven to be faster and more accurate tools than the conventional methods [4-5].

Initially, Nocardia asteroides was assumed to be the only causative agent of nocardiosis. However, studying 78 clinical samples from Nocardia patients revealed six different antimicrobial susceptibility patterns for 12 antimicrobial agents (ampicillin, amoxicillin-clavulanic acid, ceftriaxone, linezolid, amikacin, imipenem, ciprofloxacin, clarithromycin, gentamicin, kanamycin, erythromycin, sulfamethoxazole). Later, advances in molecular sciences showed that Nocardia asteroides were a group of bacteria with high genetic similarities and were designated as Nocardia asteroides complex [6, 1]. Wallace and colleagues described six drug susceptibility pattern types among 78 clinical isolates previously identified as "Nocardia asteroides." Their work was the most significant study of antimicrobial susceptibilities of Nocardia asteroides that revealed the variability of drug susceptibility patterns among the isolates and the first to show a grouping of specific susceptibility patterns. Later in the 1990s, with the development of molecular techniques, molecular taxonomy studies revealed that Nocardia abscessus belonged to the first drug susceptibility pattern. Similarly, Nocardia brevicatena-paucivorans complex was categorized in the second drug susceptibility pattern, Nocardia nova complex (Nocardia nova, Nocardia veterana, Nocardia africana, and Nocardia kruczakiae) in the third drug susceptibility pattern, Nocardia transvalensis complex (N. transvalensis sensu stricto, Nocardia wallacei, and Nocardia blacklockiae) in the fourth drug susceptibility pattern, and Nocardia farcinica and Nocardia cyriacigeorgica in the fifth and sixth patterns of drug susceptibility, respectively [1]. According to the difference in the susceptibility pattern of each member of the Nocardia asteroides complex, it can be concluded that prescription of effective antibiotics should be after the accurate identification of the causative Nocardia species. Also, clinical studies showed that some Nocardia species such as Nocardia otitidiscaviarum and Nocardia beijingensis could cause systematic infections, while other species like Nocardia brasiliensis cause limited local infections $[1,6-7]$. Trimethoprim-sulfamethoxazole, amikacin, and imipenem are usually the first drug choices for nocardiosis [7]. However, the rate of fatality among patients with pulmonary and brain abscess who consumed trimethoprim-sulfamethoxazole ranged from 20 to 50\% [8].

${ }^{*}$ Correspondence: Masoud Keikha

Department of Microbiology, School of Medicine, Isfahan Medical University, Isfahan, Iran, 7346181746

Email: masoudkeikha@outlook.com

Tel: +98 (938) 6836425 Fax: +98 (31) 37929038 
Also, some patients are allergic to sulfonamide drugs, which necessitates the performance of drug susceptibility test for the Nocardia species isolated from the clinical specimens. According to CLSI, the standard method for determining the drug sensitivity for this group of bacteria is microdilution broth [8-9]. All pathogenic Nocardia species showed to be susceptible to linezolid and amikacin, except members of $N$. transvalensis complex [8]. Also, another similar study demonstrated susceptibility of clinical isolates of Nocardia to linezolid and their minimal resistance to trimethoprim-sulfamethoxazole. While Nocardia pseudobrasiliensis and $N$. transvalensis complex, as the most common species, were resistant to trimethoprimsulfamethoxazole [10].

In summary, due to differences in the pattern of drug susceptibility among clinical isolates of the Nocardia species, especially members of complex species, e.g., Nocardia asteroids, accurate identification of Nocardia species using reliable molecular tools such as sequencing of $16 \mathrm{~S}$ rRNA fragment or $h s p$-RFLP is necessary for appropriate and successful treatment of nocardiosis.

\section{ACKNOWLEDGEMENT}

The author would like to appreciate the faculty members of Department of Microbiology, Isfahan University of Medical Sciences.

\section{CONFLICT OF INTEREST}

The authors declare that there are no conflicts of interest associated with this manuscript.

\section{REFERENCES}

1. Brown-Elliott BA, Brown JM, Conville PS, Wallace RJ. Clinical and laboratory features of the Nocardia spp. based on current molecular taxonomy. Clin Microbiol Rev. 2006; 19 (2): 259-82.

2. Menkü A, Kurtsoy A, Tucer B, Yıldız O, Akdemir H. Nocardia brain abscess mimicking brain tumour in immunocompetent patients: report of two cases and review of the literature. Acta Neurochir (Wien). 2004; 146 (4): 411-14.

3. Rahdar HA, Azadi D, Shojaei H, Daei-Naser A. Molecular analysis and species diversity of Nocardia in the hospital environment in a developing country, a potential health hazard. J Med Microbiol. 2017; 66 (3): 334-41.

4. Bafghi MF, Heidarieh P, Habibnia S, Rasouli-Nasab M, Kalantar Neyestanaki D, Afshar D, et al. Phenotypic and molecular properties of the Nocardia species. Avicenna J Clin Microb Infect. 2014; 1 (1): e19215.

5. Rudramurthy SM, Honnavar P, Kaur H, Samanta P, Ray P, Ghosh A, et al. Molecular identification of clinical Nocardia isolates from India. J Med Microbiol. 2015; 64 (10): 1216-25.

6. Welsh O, Vera-Cabrera L, Salinas-Carmona MC. Current treatment for Nocardia infections. Expert Opin Pharmacother. 2013; 14 (17): 2387-98.

7. Taj-Aldeen SJ, Deshmukh A, Doiphode S, Abdul Wahab A, Allangawi M, AlMuzrkchi A, et al. Molecular identification and susceptibility pattern of clinical Nocardia species: Emergence of Nocardia crassostreae as an agent of invasive nocardiosis. Can J Infect Dis Med Microbiol. 2013; 24 (2): 33-8.

8. Wallace RJ, Steele LC. Susceptibility testing of Nocardia species for the clinical laboratory. Diagn Microbiol Infect Dis. 1988; 9 (3): 155-66.

9. McTaggart LR, Doucet J, Witkowska M, Richardson SE. Antimicrobial susceptibility among clinical Nocardia species identified by multilocus sequence analysis. Antimicrob Agents Chemother. 2015; 59 (1): 269-75.

10. Schlaberg R, Fisher MA, Hanson KE. Susceptibility profiles of Nocardia isolates based on current taxonomy. Antimicrob Agents Chemother. 2014; 58 (2): 795-800. 